\title{
حول توزيع الطلب خلال فترة الانتظار عند خضوع الطلب لتوزيع كَاها وفترة الانتظار للتوزيع اللوغاريتمي الطبيـي
}

الأستاذ الاكتور صلاح حمزة عبد الإحماء

قسم الإحصاء/ الجامعة المستتصرية

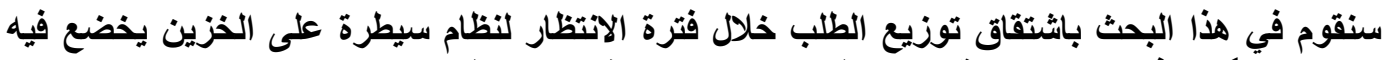

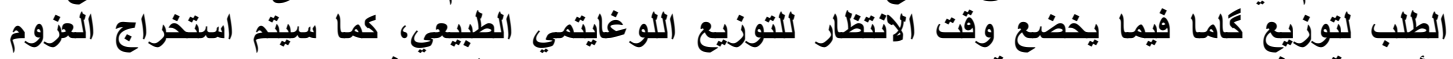

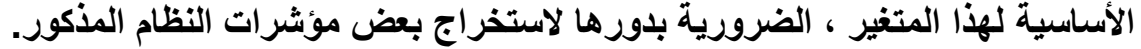

المصطلحات المستخدمة: التكامل المحيط، المستوي المركب، تكامل هانكيل، مستوى إعادة الطلب، الوقاية. (القدمثة

قـ يكون من الضروري في بعض التطبيقات الإحصائية معرفة بعض خواص مجموع مجموعة من

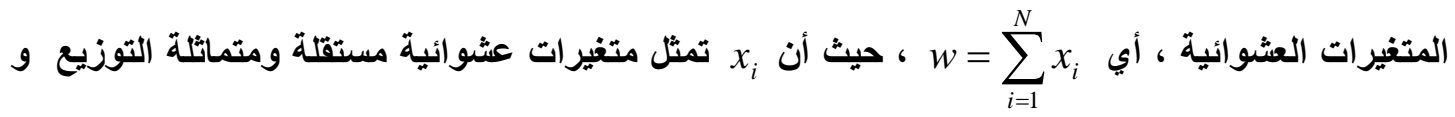
N عبارة عن متغير عشوائي أيضا" مستقل عن x

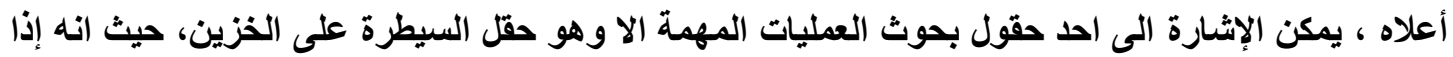

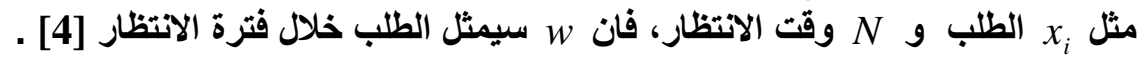

ان الخواص الاحصائية للمتغير w ، وبشكل خاص العزوم ، يمكن الحصول عليها بسهولة من خلال افتراض ان ان الن

$$
\begin{aligned}
M_{w}(t) & =E\left[M_{x i}(t)\right]^{N} \\
& =P_{N}\left[M_{x i}(t)\right]
\end{aligned}
$$
P $P_{N}(t)$

ومن خلال المعادلة (1) اعلاه ، يمكن الحصول على توقع وتباين المتغير w بالثكل [1] ،

$$
\left.\begin{array}{l}
E(w)=E(x) . . E(N) \\
\operatorname{Var}(w)=[E(x)]^{2} \operatorname{Var}(N)+E(N) \operatorname{Var}(x)
\end{array}\right\}
$$

والمعادلتين الاخيرتين في (Y) اعلاه تعتبران الاساس في تحديد نقطة اعادة الطلب Reorder Level في 


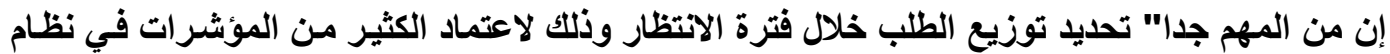

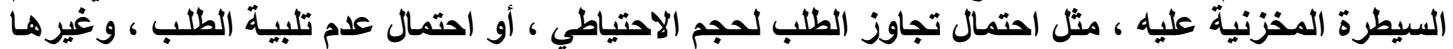

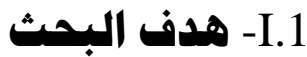

لنفترض ان توزيع الطب هو (x) ، $0 \leq N \leq N^{*}$

$\int_{0}^{N^{*}} f(x)_{N} g(N) d N$

حيث ان

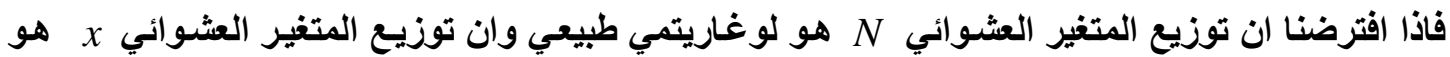

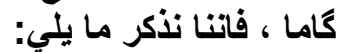

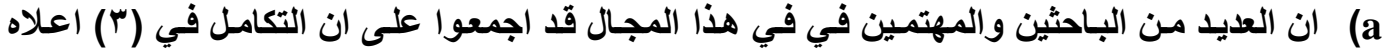

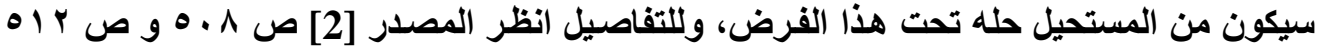

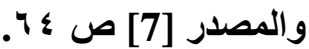

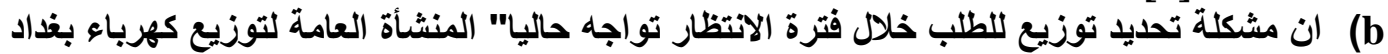

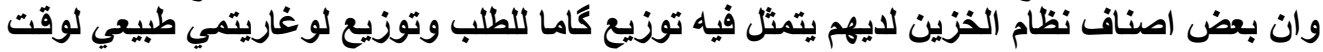
الانتظار .

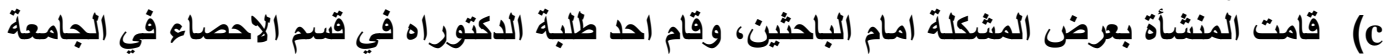

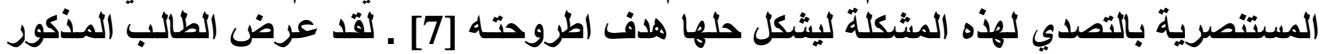

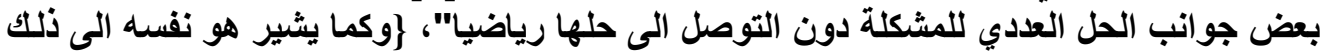

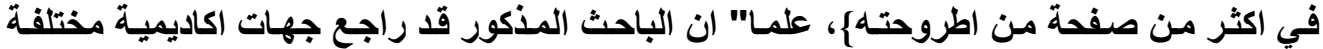

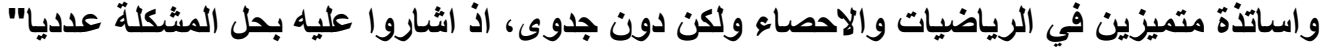

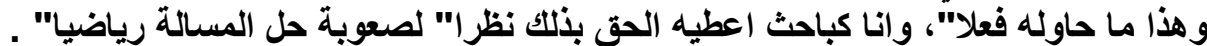

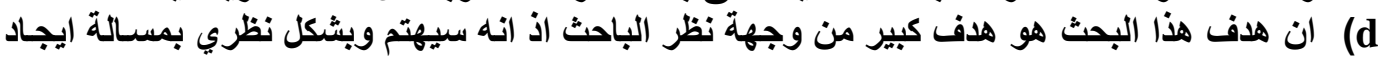

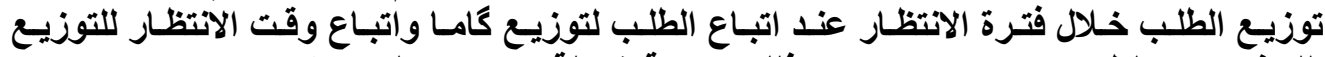

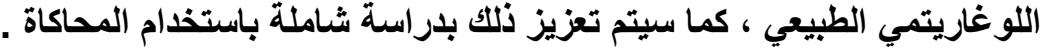

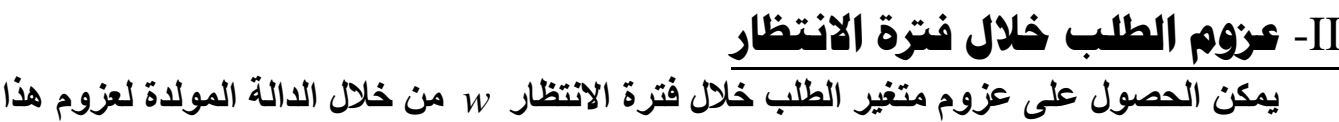
المتغير والتي سنستخرجها من خلال النظرية التالية ،

الدالـة المولدة لعزوم المتفير العثـوائي

نظرية

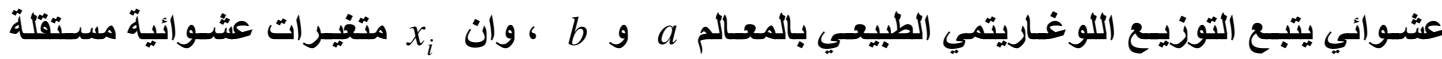

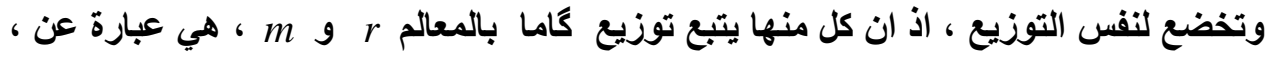
$M_{w}(h)=\sum_{j=0}^{\infty} \frac{(-r)^{j}}{j !}[\operatorname{Ln}(1-h / m)]^{j} e^{j a+j^{2} b / 2}$ 


$$
\begin{aligned}
& P_{N}(t)=E\left(t^{N}\right)=\int_{-\infty}^{\infty} t^{e^{y}} \frac{e^{\frac{-(y-a)^{2}}{2 b}}}{\sqrt{2 \pi b}} d y \\
& =\int_{-\infty}^{\infty} e^{e^{y} \operatorname{Ln}(t)} \frac{e^{\frac{-(y-a)^{2}}{2 b}}}{\sqrt{2 \pi b}} d y=\int_{-\infty}^{\infty} \sum_{j=0}^{\infty} \frac{\left(e^{y} \operatorname{Ln}(t)\right)^{j}}{j !} \cdot \frac{e^{\frac{-(y-a)^{2}}{2 b}}}{\sqrt{2 \pi b}} d y \\
& =\sum_{j=0}^{\infty} \frac{(\operatorname{Ln}(t))^{j}}{j !} \int_{-\infty}^{\infty} e^{y j} \frac{e^{\frac{-(y-a)^{2}}{2 b}}}{\sqrt{2 \pi b}} d y=\sum_{j=0}^{\infty} \frac{(\operatorname{Ln}(t))^{j}}{j !} e^{j a+j^{2} b / 2} \\
& \text { و ويما ان } \\
& M_{w}(h)=P_{N}\left(M_{x}(h)\right)=\sum_{j=0}^{\infty} \frac{\left[\operatorname{Ln}(1-h / m)^{-r}\right]^{j}}{j !} e^{j a+j^{2} b / 2} \\
& \text { ومنها تنتج المعادلة (؛) ، وبذلك نكون قد اتممنا برهان النظرية . }
\end{aligned}
$$




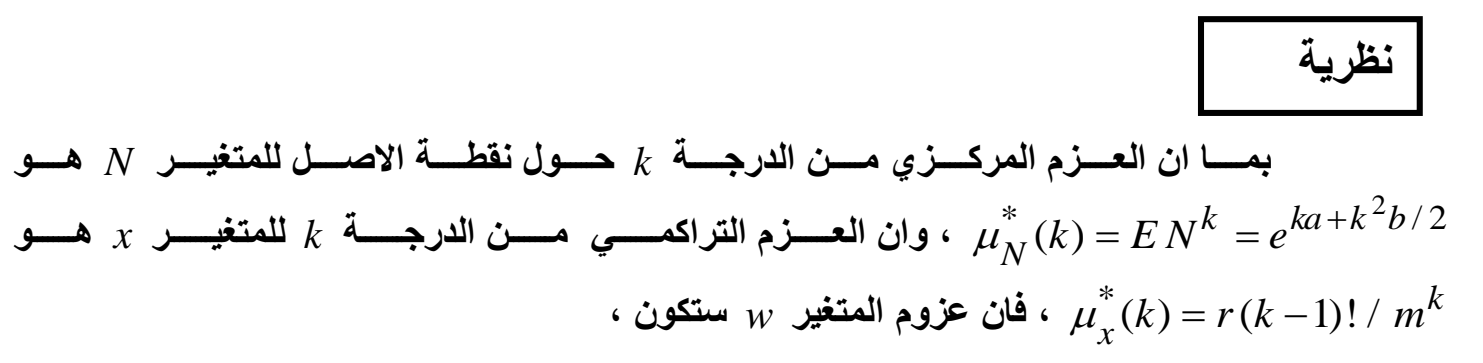

(a)

$$
\begin{gathered}
M_{w}^{\prime}(h)=\sum_{j=0}^{\infty} \frac{(-r)^{j}}{j !} j[\operatorname{Ln}(1-h / m)]^{j-1} \frac{e^{j a+j^{2} b / 2}}{1-h / m} \cdot \frac{-1}{m} \\
\Rightarrow E(w)=\mu_{w}^{*}(1)=M_{w}^{\prime}(0)=(r / m) e^{a+b / 2} \\
=E(x) \cdot E(N)=\mu_{N}^{*}(1) \cdot \mu_{x}^{*}(1)
\end{gathered}
$$

(b)

$$
\begin{aligned}
& M_{w}^{\prime /}(h)=\sum_{j=0}^{\infty} \frac{-(-r)^{j}}{m j !} j e^{j a+j^{2} b / 2}\left\{\frac{1}{m}[\operatorname{Ln}(1-h / m)]^{j-1}(1-h / m)^{-2}-\frac{(j-1)[(1-h / m)]^{j-2}}{m \cdot(1-h / m)^{2}}\right\} \\
& \text { ويذلك وعند j=1 للحد الاول و j=2 للحد الثاني ، فانه يكون ، } \\
& M_{w}^{\prime \prime}(0)=E\left(w^{2}\right)=\mu_{w}^{*}(2)=\frac{r}{m^{2}} \cdot e^{a+b / 2}+\frac{r^{2}}{m^{2}} \cdot e^{2 a+2 b} \\
& =\operatorname{Var}(x) \cdot E(N)+(E(x))^{2} \cdot E\left(N^{2}\right) \\
& \Rightarrow \operatorname{Var}(w)=\operatorname{Var}(N)[E(x)]^{2}+\operatorname{Var}(x) \cdot E(N) \\
& \Rightarrow \quad C V_{w}\left[\frac{C V_{x}^{2}}{E(N)}+C V_{N}^{2}\right]^{1 / 2}
\end{aligned}
$$


(c) $E\left(w^{3}\right)=E\left(x-\mu_{x}\right)^{3} E(N)+3 E(x) \operatorname{Var}(x) E\left(N^{2}\right)+(E(x))^{3} E\left(N^{3}\right)$

(d)

$E\left(w^{4}\right)=E(N) \mu_{x}^{*}(4)+11[\operatorname{Var}(x)]^{2} E\left(N^{2}\right)+6 \operatorname{Var}(x) 3(E(x))^{2} E\left(N^{3}\right)+(E(x))^{4} E\left(N^{4}\right)$ $--(\mathbf{1 0})$

(e) $E\left(w^{k}\right)=\mu_{w}^{*}(k)=\frac{1}{m^{k}} \sum_{j=1}^{k} c_{j} r^{j} e^{j a+j^{2} b / 2}$

(f)

$$
\begin{aligned}
& \text { ، فان } \quad \mu_{x}^{* *}(k)=\frac{\mu_{x}^{*}(k)}{(k-1) !}=\frac{r}{m^{k}} \text { اذ كتبنا } \mu_{w}^{*}(k)=\mu_{x}^{* *}(k) \cdot \sum_{j=1}^{k} c_{j} r^{j-1} \mu_{N}^{*}(j) \quad----(12)
\end{aligned}
$$

- توزيع الطاب خلال فترة الانتظار -III

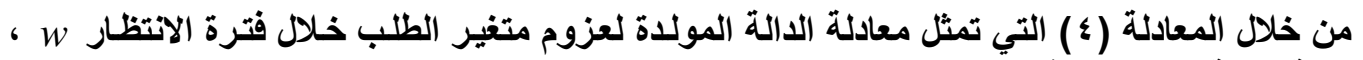
فيمكن كتابة الدالة المميزة لهذا المتغير بالثكل ،

$$
\begin{aligned}
Q_{w}(h)=E\left(e^{i h w}\right) & =M_{w}(i h) \\
& =\sum_{j=0}^{\infty} \frac{(-r)^{j}}{j !}[\operatorname{Ln}(1-i h / m)]^{j} e^{j a+j^{2} b / 2}
\end{aligned}
$$

وعليه يمكن الحصول على دالة كثافة احتمال الطلب خلال فترة الانتظار من خلال حل تكامل فورير التالي، $f(w)=\frac{1}{2 \pi} \int_{-\infty}^{\infty} e^{-i h w} Q_{w}(h) d h$ 


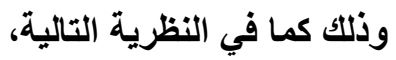

\section{نظرية}

اذا كاتت المعادلة (ب ا ) تمثل الادالة المميزة للمتغير w ، فان دالة كثافة احتمال هذا المتغير ستكون، $f(w)=\frac{1}{w} \sum_{j=1}^{\infty} \frac{r^{j}}{j !} e^{j a+j^{2} b / 2}\left[\sum_{s_{1}=1}^{\infty} \ldots \sum_{s_{j}=1}^{\infty} \frac{\left(\sum_{\ell=1}^{j} s_{\ell}\right) !}{(w m)^{\sum s_{\ell}} \prod_{\ell=1}^{j} s_{\ell}}\right]$

البر هان

\section{باستخدام الصيغة رقم (؛ (1) يكون ،}

$f(w)=\frac{1}{2 \pi} \int_{-\infty}^{\infty} e^{-i h w} d h+\frac{1}{2 \pi} \int_{-\infty}^{\infty} e^{-i h w} \sum_{j=1}^{\infty} \frac{(-r)^{j}}{j !}[\operatorname{Ln}(1-i h / m)]^{j} e^{j a+j^{2} b / 2} d h$

$=\frac{1}{2 \pi} \int_{-\infty}^{\infty} e^{-i h w} d h+\frac{1}{2 \pi} \sum_{j=1}^{\infty} \frac{(-r)^{j}}{j !} e^{j a+j^{2} b / 2} \int_{-\infty}^{\infty} e^{-i h w}[\operatorname{Ln}(1-i h / m)]^{j} d h$

وباستخدام العلاقة

$f(w)=\frac{1}{2 \pi} \int_{-\infty}^{\infty} e^{-i h w} d h+\frac{1}{2 \pi} \sum_{j=1}^{\infty} \frac{(-r)^{j}}{j !} e^{j a+j^{2} b / 2} \int_{-\infty}^{\infty} e^{-i h w}\left[\sum_{k=1}^{\infty} \frac{(h i / m)^{k}}{k}\right]^{j} d h$

، و $\left[\sum_{k=1}^{\infty} \frac{(h i / m)^{k}}{k}\right]^{j}=\sum_{s_{1}=1}^{\infty} \ldots \sum_{s_{j}=1}^{\infty} \frac{(h i / m)^{\sum_{\ell=1}^{j} s_{\ell}}}{\prod_{\ell=1}^{j} s_{\ell}}$ ، فيما ان

$f(w)=\frac{1}{2 \pi} \int_{-\infty}^{\infty} e^{-i h w} d h+\frac{1}{2 \pi} \sum_{j=1}^{\infty} \frac{r^{j}}{j !} e^{j a+j^{2} b / 2} \sum_{s_{1}=1}^{\infty} \ldots \sum_{s_{j}=1}^{\infty} \frac{(-1 / m)^{\sum_{\ell=1}^{j} s_{\ell}}}{\prod_{\ell=1}^{j} s_{\ell}} \int_{-\infty}^{\infty} e^{-i h w}(-i h)_{\ell=1}^{j} s_{\ell} d h$ 


\section{ان حدود التكامل في الدالة اعلاه يمكن احتسـابها باستخدام التكامل المحيط counter integration في} المستوي المركب complex plane وذلك بعد اخذ التحويل التهابل

$$
z=i h w \quad \Rightarrow h=z / i w \quad, \quad d h=(1 / i w) d z \quad-----(16)
$$

$$
\begin{aligned}
& f(w)=\frac{1}{w} \frac{i}{2 \pi} \int_{-\infty}^{\infty} e^{-z} d z+\sum_{j=1}^{\infty} \frac{r^{j}}{j !} e^{j a+j^{2} b / 2} \sum_{s_{1}=1}^{\infty} \ldots \sum_{s_{j}=1}^{\infty} \frac{(-1 / m)^{\sum_{\ell=1}^{j} s_{\ell}}}{\prod_{\ell=1}^{j} s_{\ell}} w^{-\left(\sum s_{\ell}+1\right)} \frac{i}{2 \pi} \int_{+i \infty}^{-i \infty} e^{-z}(-z)_{\ell=1}^{\sum_{\ell}^{j} s_{\ell}} d z \\
& \frac{i}{2 \pi} \int_{+i \infty}^{-i \infty} e^{-z}(-z)_{\ell=1}^{j} s_{\ell} d z=\frac{1}{\left(-\sum_{\ell=1}^{j} s_{\ell}-1\right) !} \\
& =\frac{\left[\sum_{\ell=1}^{j} s_{\ell}\right] !}{(-1)^{\sum_{\ell=1}^{j} s_{\ell}+2}} \\
& \text { وحيث ان ، }
\end{aligned}
$$

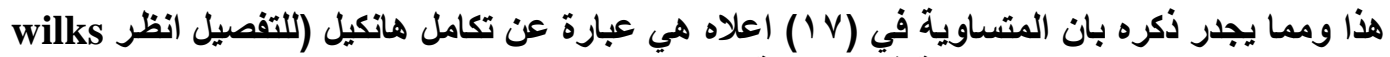

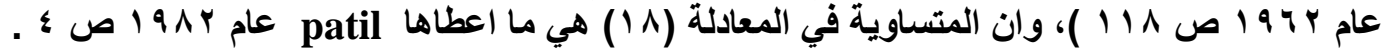

$$
\begin{aligned}
& \text { نتيجة } \\
& \text { - } w \cong G a m m a\left(r e^{a}, m\right) \text { عندما } b \rightarrow 0 \text { فان } \\
& \text { البر هان } \\
& Q_{w}(h) \cong \sum_{j=0}^{\infty} \frac{\left[-r e^{a} \operatorname{Ln}(1-i h / m)\right]^{j}}{j !}
\end{aligned}
$$

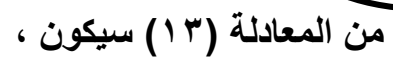

$$
\begin{aligned}
& =e^{-r e^{a} \operatorname{Ln}(1-i h / m)}=[1-i h / m]^{-r e^{a}}
\end{aligned}
$$

وبذلك نكون قد أتممنا برهان النتيجة . 


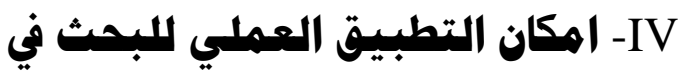

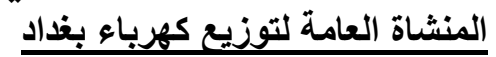

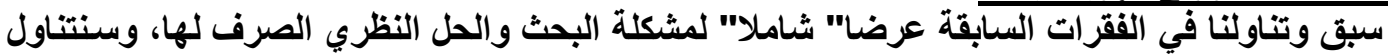

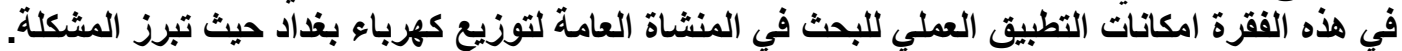

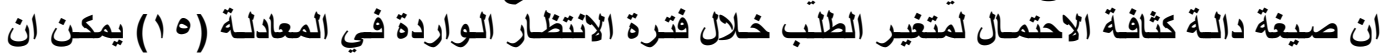

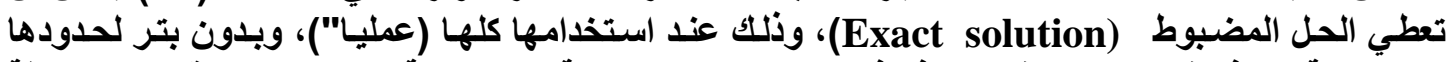
اللامتناهية، وهذا غير ممكن وغير متوفر في الحاسبات الاكترونية المستخدمة حاليا" على الاقل، وتلك نقطة

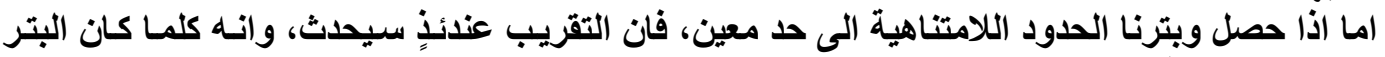

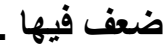

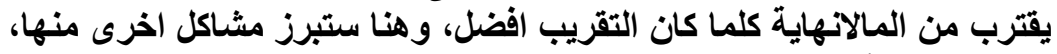

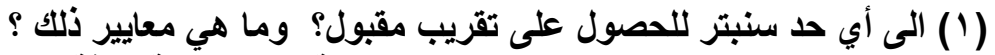
(Y) الوقت الكبير المستغرق للحصول على على كل قيمة من قيم دئ دالة كثافة الاحتمال.

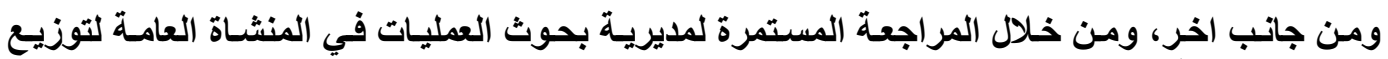

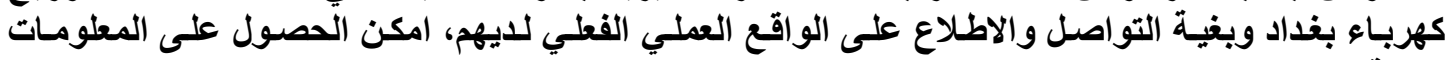

(1) ان الانحراف المعياري للطلب الفصلي (Quarterly demand) يكون دالة بدلالة متوسط الطلب الفصـلي

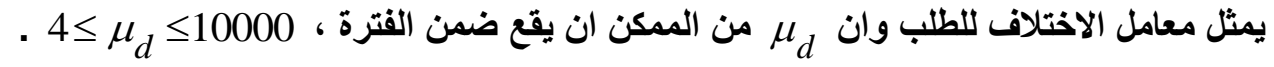

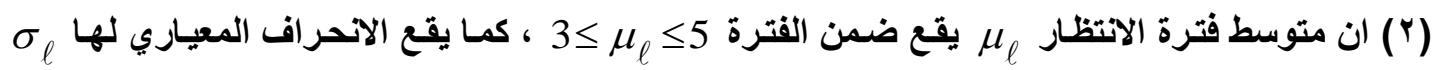
ضمن الفترة $1.5 \leq \sigma_{\ell}$ ض 1.5

$$
\begin{aligned}
& \text { وحيث ان فترة الانتظار تتبع التوزيع اللوغاريتمي الطبيعي فانه يمكن كتابة ، } \\
& \mu_{e}=e^{a+b / 2} \\
& \mu_{\ell}^{2}+\sigma_{l}^{2}=e^{2 a+2 b} \\
& b=\operatorname{Ln}\left(\mu_{\ell}^{2}+\sigma_{\ell}^{2}\right)-\operatorname{Ln}\left(\mu_{\ell}^{2}\right) \\
& =\operatorname{Ln}\left[\frac{\sigma_{\ell}^{2}}{\mu_{\ell}^{2}}+1\right]
\end{aligned}
$$

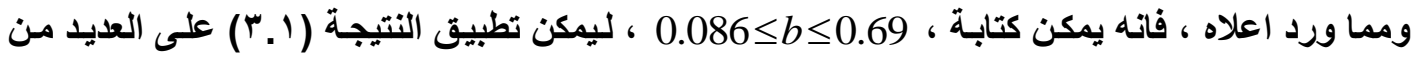

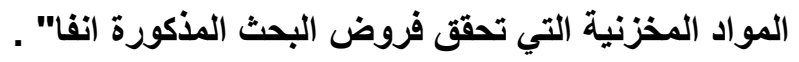


1) Burgin , T.A. (1972) "Inventory control with Normal demand and Gamma lead times" ; Op.res.Quart., Vol.23, No.1, March , PP.73-81 .

2) (1975) " The Gamma distribution and inventory control ";

Op. Res. Quart. Vol.26, No.3, September, PP.507-527 .

3) Patil, G.P. \& Boswell, M.T. \& Joshi , S.W. and Ratnaparkhi, M.V. (1984)

"Dictionary Of statistical distributions "; Vol.1 : Discrete Models . ICPH Series .

4) Ray , W.D. (1980) " The significance of correlated demands and variable lead times for stock control policies "; J. Opl. Res. Soc., Vol.31 , PP.187-190 . 5) (1981) " computation of reorder levels when the demands are correlated and the lead time random "; J. Opl. Res. Soc., Vol.32, PP.27-31.

6) Wilks, S. (1962) " Mathematical Statistics “, Wiley series, USA .

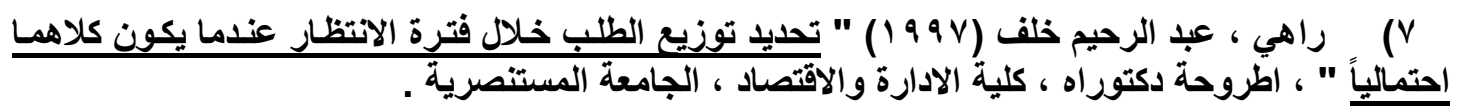

